\title{
On a Model Based Practical Control Algorithm
}

\author{
Vasile CIRTOAJE*, Alina BAIESU \\ Department of Automatic Control and Computers, \\ Petroleum and Gas University of Ploiesti, \\ 39 Bucuresti Avenue, 100680, Ploiesti, Romania \\ vcirtoaje@upg-ploiesti.ro (*Corresponding author), agutu@upg-ploiesti.ro
}

\begin{abstract}
The design of the proposed algorithm relies on three basic ideas: (1) finding a model-based controller so that for any stable process of proportional type, the closed-loop controller output to a step reference has a step shape (or close to this form) and removes the steady-state error; (2) refining the controller structure so that the initial value of the controller output to a step reference is $K$ times its final value, where $K$ is a tuning parameter with standard value 1 ; (3) extending the controller structure to address integral processes and some unstable processes by turning them into stable compensated processes of proportional type. The overall controller is a series connection P-IMC of two systems: one of pure proportional type and another one of IMC type. There is a simple procedure to verify online if the model parameters (steady-state gain, time delay and transient time) have suitable values and to adjust them to improve the model. As the PID control algorithm, the proposed method is quasi-universal and practical, but it is superior by its control performance and the simplicity of the tuning procedure (which enables poorly trained workers to easily operate the control system). Also, it is more practical than the classical IMC algorithm because its equations have a unique form for all process types (as the PID algorithm), and has a control gain as tuning parameter instead of a filter time constant. Several applications are given to show the effectiveness of the algorithm for different types of process.
\end{abstract}

Keywords: Practical and quasi-universal algorithm, Model based algorithm, Simple online tuning, Transient time, Time delay, Proportional process, Integral process, Unstable process, Compensated process.

\section{Introduction}

Due to the structure simplicity and the capability to control almost all processes, the PID controllers are the most widely used in industrial applications despite the recent advances in information technology and computer science (Marlin, 1995; Brosilow and Joseph, 2002; Liu et al., 2005; Chia and Lefkowitz, 2010; Visioli and Zhong, 2011, Jin and Liu, 2014). For many complex processes (especially with time delay, with overshoot or of nonminimum phase), the PID controllers cannot achieve a very good control performance. In addition, there is not a simple and intuitive method of controller tuning (Ziegler and Nichols, 1942; Garcia and Morari, 1982; Duma et al., 2011; Nicolau, 2013; Singh et al., 2014). The proposed control algorithm is inspired from the internal model control (IMC) concept, which states that an accurate control can be achieved if the control system encapsulates a representation of the controlled process (Francis and Wonham, 1976; Bengtsson, 1977; Garcia and Morari, 1982; Rivera et al., 1986; Horn et al., 1996).

In any IMC structure, the process and its model are connected in parallel so that the difference between their outputs comes back to the internal controller. The overall controller of an IMC structure is parameterized as follows

$G_{C}(s)=\frac{Q(s)}{1-Q(s) G_{M}(s)}$,

https://doi.org/10.24846/v27i1y201809 where $G_{M}(s)$ is the transfer function of the process model and $Q(s)$ is the transfer function of the internal controller. In the IMC strategy, $Q(s)$ is an approximate inverse of the model transfer function, which includes a suitable filter to guarantee the controller properness. The filter time constant is the main tuning parameter of the overall controller.

Despite its advantages, the IMC strategy did not become a strong practical alternative to the PID strategy (Normey and Camacho, 2007; Saxena and Hote, 2012; Vanavil et al., 2014; Cirtoaje, 2017) because of the multitude of model variants that depend on the process type (linear or nonlinear, with or without overshoot, with or without oscillations, of minimum or nonminimum phase, of proportional or integral type, stable or unstable etc.). The proposed control algorithm removes this disadvantage by using a unique model structure which is associated either with the original process to be controlled (if it is stable and of proportional type) or with the compensated process (if the original process is of integral type or unstable).

In the last years, many publications have presented various tuning techniques of IMC based PID controllers for different types of process (Brosilow, 2002; Nageswara and Padma, 2010; Jin and Liu, 2014; Singh et al., 2014; Vanavil et al., 2014; Santosh and Padma, 2016; Ghousiya et al., 2017). In our opinion, the control performance 
derived by using IMC based PID algorithms is generally weaker that the one obtained by using control algorithms of genuine IMC type.

The general structure of a linear closed-loop control system is shown in Figure 1, where $G_{P}$ is the process transfer function, $G_{C}$ - controller transfer function, $Y$ - controlled variable, $U$ - control (manipulated) variable, $R$ - reference (setpoint), $E$ - the control error and $V$ - the load disturbance.

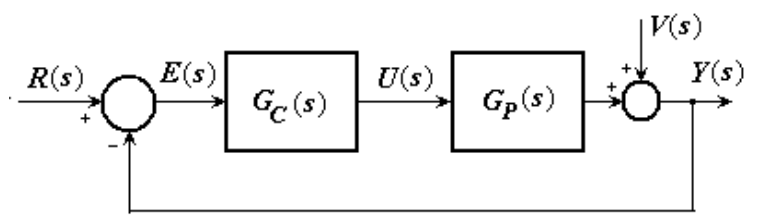

Figure 1. Block diagram of a closed-loop control system

The transfer functions between the controlled variable $Y$ and the input variables $R$ and $V$ are given by

$G_{Y R}=\frac{G_{C} G_{P}}{1+G_{C} G_{P}}, \quad G_{Y V}=\frac{1}{1+G_{C} G_{P}}$.

From

$G_{Y R}(s)+G_{Y V}(s)=1$,

it follows that the response $y(t)$ to a unit step reference and the response $y_{v}(t)$ to a unit step disturbance added to the process output are complementary:

$y(t)+y_{v}(t)=1$.

According to (4), it is not necessary to analyze the control performance of the closed-loop system to a step disturbance; it suffices to determine only the response $y(t)$ to a step reference.

The design of the proposed algorithm starts from the idea to find a model-based control structure so that, for any process of P1-type (whose input step response is monotone and finite), the control response $u(t)$ to a step reference has a step shape (or close to this form) and removes the steadystate error. Then, this structure is connected in series with a proportional block with the gain $K$ , so that the controller response to a step reference has the initial value $K$ times its final value. In this found form (called primary or standard form), the designed algorithm can be applied to control any stable proportional process (with finite and nonzero steady-state gain). Using the compensated process concept (Cirtoaje, 2006; Yamada, 2006; Nguyen et al., 2013; Vazquez Guerra, 2016), the control algorithm is extended to control integraltype processes and unstable processes. Thus, the extended form of the proposed algorithm is a quasi-universal P-IMC (proportional-internal model control) algorithm, which has the capability to control almost all process types. In addition, the model parameters can be online verified and adjusted to improve the model accuracy.

The structure and the form of the controller equations don't depend on the process to be controlled. This is possible because the embedded model addresses the compensated process, which is always stable and of proportional type.

A control gain with standard value 1 is used as tuning parameter, which is more agreeable for the process human operator than the filter time constant from the usual IMC algorithms.

Six applications in MATLAB/SIMULINK environment are presented for the main types of processes to show the tuning procedure, the control performance and the robustness to the model uncertainty.

The paper is arranged as follows. Section 2 presents the primary form of the proposed algorithm, which can be applied to control stable proportional processes with or without time delay, overshoot and oscillations, of minimum or nonminimum phase. Section 3 extends the control algorithm to integral processes and to some unstable processes. In addition, four applications are given in Section 2, and two applications in Section 3. Concluding observations and future research project are presented in Section 4.

\section{Primary form of the control algorithm}

The purpose of this section is to present the theoretical fundamentals and some practical results of the primary variant of the proposed control algorithm, and how this variant can be used to control stable proportional processes with or without time delay, overshoot and oscillations, of minimum or nonminimum phase. 


\subsection{Theoretical basis}

The transfer function between the control variable $U$ and the reference input $R$ (see Figure 1) is given by

$$
G_{U R}(s)=\frac{G_{C}(s)}{1+G_{C}(s) G_{P}(s)} .
$$

Consider first a stable proportional process with the steady-state gain $K_{P}$, and assume that the closedloop system response $u(t)$ to a unit step reference is a step function of magnitude $1 / K_{P}$. This condition (that involves zero steady-state error), equivalent to

$$
G_{U R}(s)=\frac{1}{K_{P}}
$$

and

$$
G_{C}(s)=\frac{1}{K_{P}-G_{P}(s)},
$$

leads to the following practical controller

$$
G_{C}(s)=\frac{K}{K_{M}-G_{M}(s)},
$$

where $G_{M}(s)$ is the transfer function of the process model, $K_{M}$ - model steady-state gain, $K$ - tuning gain with standard value 1 (that can be used by the process operator to make the control action stronger or weaker). Because $K_{M}=G_{M}(0)$ , the controller transfer function (8) has a pole at the origin, therefore the controller is of integraltype. Consequently, if the closed-loop system is stable, then it has zero steady-state error to a step reference or disturbance. The form below of the controller transfer function (8),

$$
G_{C}(s)=K \cdot \frac{\frac{1}{K_{M}}}{1-\frac{G_{M}(s)}{K_{M}}},
$$

leads to an algorithm structure of P-IMC type (see Figure 2), that is a series connection between a pure proportional element and a closed-loop IMC structure. If the ratio $K / K_{M}$ is constant, then the process response $y(t)$ to a step reference remains unchanged for any set value of the model gain $K_{M}$.

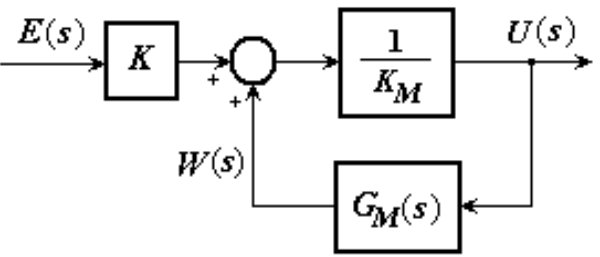

Figure 2. Primary structure of the control algorithm

A second-order model with double lag time constant plus time delay has the transfer function

$$
G_{M}(s)=\frac{K_{M} e^{-\tau_{M} s}}{\left(T_{M} s+1\right)^{2}}
$$

Such a model can describe with sufficient accuracy a P1-type process (with monotone and bounded step response). In our opinion, a firstorder model is too simple and cannot describe the process sluggishness, while a second-order model with two distinct lag time constants or a third order model are too complicated to be used for increasing the model accuracy. The model parameters $K_{M}, \tau_{M}$ and $T_{M}$ can be easily determined from the process response to a step input $u$. Clearly, the experimental procedure requires the process to be in a steady-state behavior before changing the input $u$. The model time constant $T_{M}$ depends on the model transient time $T_{t r M}$ as follows:

$T_{M}=\frac{T_{t r M}}{5.83} \approx \frac{T_{t r P}}{5.83}$,

where $T_{t r P}$ is the transient time of the process response $y(t)$ to a step input $u$ (that does not include the time delay); more precisely,

$T_{t r P}=t_{1}-\tau_{P}$,

where $\tau_{P}$ is the process time delay, and $t_{1}$ is the process settling time (when the response $y$ reaches $98 \%$ of its final value).

The discrete equivalent of the model (10) has the transfer function

$G_{M}^{0}(z) \approx \frac{K_{M}(1-p)^{2} z^{-1-l_{M}}}{\left(1-p z^{-1}\right)^{2}}, \quad p=e^{-T / T_{M}}$,

where $T$ is the sampling time and $l_{M}$ - the integer value of the ratio between the model time delay and the sampling time:

$l_{M}=\left[\frac{\tau_{M}}{T}\right]$ 
The initial value $u\left(0_{+}\right)$and the final value $u(\infty)$ of the closed-loop system response $u(t)$ to a unit step reference are

$$
u\left(0_{+}\right)=\frac{K}{K_{M}}, \quad u(\infty)=\frac{1}{K_{P}} .
$$

In addition, the response $u(t)$ keeps its initial value $K / K_{M}$ on the time interval $\left[0, \tau_{M}\right]$. By increasing/decreasing the tuning gain $K$, the process operator can make the control action stronger/weaker.

For $K=1$, the initial and final values of the control response $u(t)$ to a unit step reference are $u\left(0_{+}\right)=1 / K_{M}, u(\infty)=1 / K_{P}$. If $K_{M}=K_{P}$, then the initial value $u\left(0_{+}\right)$and the final value $u(\infty)$ are equal. Moreover, for $K=1$ and a perfect model with $G_{M}(s)=G_{P}(s)$, the response $u(t)$ is a step function of magnitude $1 / K_{P}$, i.e.

$u(t)=\frac{1}{K_{P}} \cdot 1(t)$,

where $1(t)$ is the unit step function. Since the process model is not perfect, the response $u(t)$ to a step reference change $\Delta r$ is not a perfect step function. Assume further that $K=1, K_{P}>0$ and $\Delta r>0$.

If the model steady-state gain $K_{M}$ differs from the process steady-state gain $K_{P}$, then the closedloop system response $u(t)$ to a step reference has the initial value $u\left(0_{+}\right)$different from its steadystate value $u(\infty)$; more precisely, $u\left(0_{+}\right)>u(\infty)$ for $K_{M}<K_{P}$, and $u\left(0_{+}\right)<u(\infty)$ for $K_{M}>K_{P}$ (see Figure 3).

If the model time delay $\tau_{M}$ differs from the process time delay $\tau_{P}$, then $u(t)$ has a deviation from the step form immediately after the time $t_{0}$, where $t_{0}=\min \left\{\tau_{P}, \tau_{M}\right\}$. This deviation is positive for $\tau_{M}<\tau_{P}$ and negative for $\tau_{M}>\tau_{P}$ (see Figure 4).

For $K_{M} \approx K_{P}$ and $\tau_{M} \approx \tau_{P}$, if the model transient time $T_{t r M}$ differs from the process transient time $T_{t r P}$, then $u(t)$ has a deviation from the step form in a time zone with $t>\tau_{M}$. The deviation is positive for $T_{t r M}<T_{t r P}$ and negative for $T_{t r M}>T_{t r P}$ (see Figure 5).

In conclusion, if the closed-loop system response $u(t)$ to a step reference is larger/smaller than the ideal step form of magnitude $1 / K_{P}$, then the respective parameter of the model need to be suitably increased/decreased.

Even if $K_{M}, \tau_{M}$ and $T_{t r M}$ have the best values, the model (10) is not perfect, therefore the response $u(t)$ has not a perfect step form, but is both above and below the step graph so that the maximum deviation from the step graph is minimum.

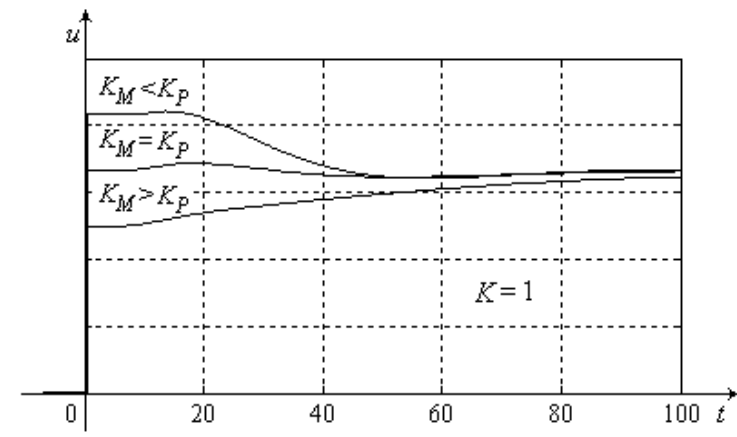

Figure 3. Closed-loop system responses $u(t)$ to a step reference for various values of $K_{M}$

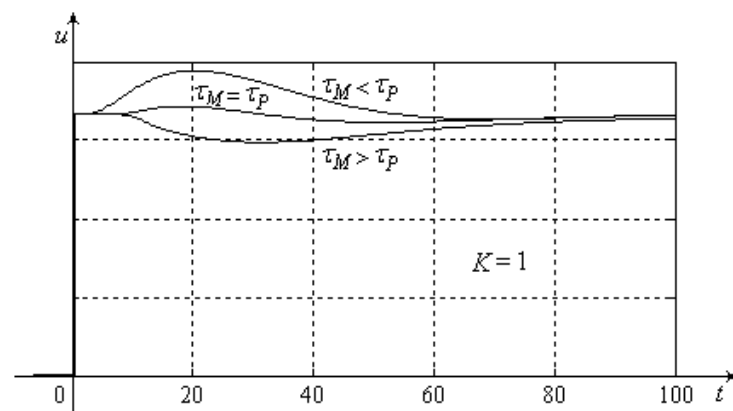

Figure 4. Closed-loop system responses $u(t)$ to a step reference for various values of $\tau_{M}$

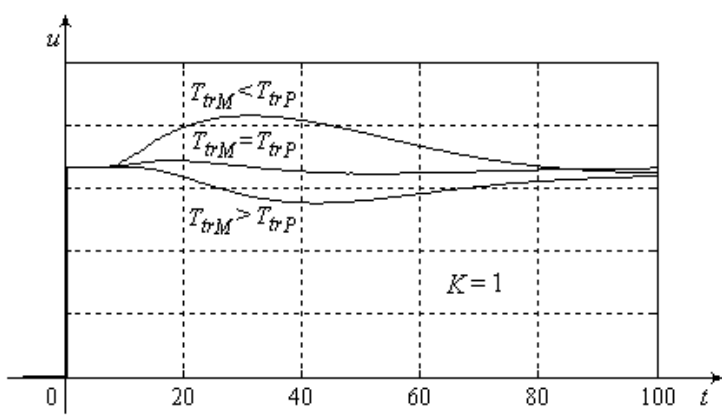

Figure 5. Closed-loop system responses $u(t)$ to a step reference for various values of $T_{t r M}$

By studying carefully the response $u(t)$ to a step reference for $K=1$, the process operator can adjust online the model parameters to improve the process model. First, he adjusts the model 
steady-state gain $K_{M}$ so that the initial value and the final value of $u$ become close to each other, then the model time delay $\tau_{M}$ and, finally, the model transient time $T_{t r M}$ - so that the deviation of $u$ from the step form in the transient zone of the response is minimum. In practical applications, to have a good control performance, it is not necessary to set the best values of the process model (10); it suffices to have only an approximate model.

For a suitable model, the closed-loop system responses $u(t)$ to a reference step for different values of the controller gain $K$ are shown in Figure 6. If $K$ is larger/smaller, then the system response $u$ is also larger/smaller on the first time interval, and the control action is stronger/weaker. In addition, the response $u$ holds its initial value $K / K_{M}$ on the time interval $\left[0, \tau_{M}\right]$. For this reason, a large $K$ provides a strong control action for processes with large time delay. As a consequence, the system response $u$ to a reference step is never very sharp (as in the case of the IMC algorithms), and a lowpass reference filter is not necessary.

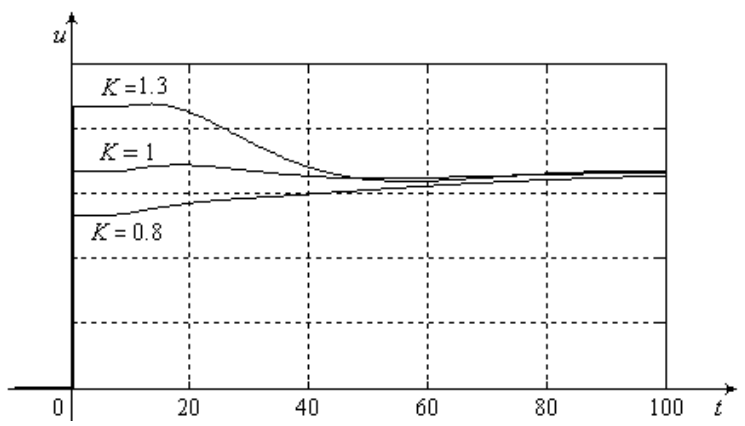

Figure 6. Closed-loop system responses $u(t)$ to a step reference for various values of $K$

Remark 2.1. The control algorithm can also be used to control stable proportional processes of nonminimum phase. For this, it suffices to consider that the process time delay $\tau_{P}$ covers the time interval where the sign of the process response to a step input is contrary to the sign of its final value.

Remark 2.2. The control algorithm can be used to control stable proportional processes with overshoot $\sigma>0$. The basic idea is to use a model steady-state gain larger than the process steady-state gain $\left(K_{M}>K_{P}\right)$, so that the control response $u$ to a step reference has a smaller initial value to reduce or to vanish the overshoot of the closed-loop system. More precisely, assuming that the step process response $y(t)$ reaches its maximum value $(1+\sigma) y(\infty)$ at the time $t_{0}$, the recommended values of the process model parameters are the following:

$K_{M}=(1+\sigma) K_{P}$,

$\tau_{M}=\tau_{P}$,

$T_{t r M}=t_{0}-\tau_{P}$.

These formulae can be obtained by approximating the process with the one whose step input response remains constant for $t \geq t_{0}$.

\subsection{Simulation results}

In this subsection, four applications for different types of linear proportional processes are presented: a process with monotone response to a step input, a process of non-minimum phase, an oscillatory process and a non-oscillatory process with overshoot.

Application 2.1. Consider the proportional process

$$
G_{P}(s)=\frac{1.5(2 s+1) e^{-6 s}}{(6 s+1)(10 s+1)(15 s+1)}
$$

whose response to a unit step input is monotone and finite (see Figure 7). From the process response, it is easy to determine the following model parameters:

$$
\begin{aligned}
& K_{M}=1.5, \\
& \tau_{M}=9, \\
& T_{t r M}=t_{1}-\tau_{P} \simeq 89-9=80 .
\end{aligned}
$$

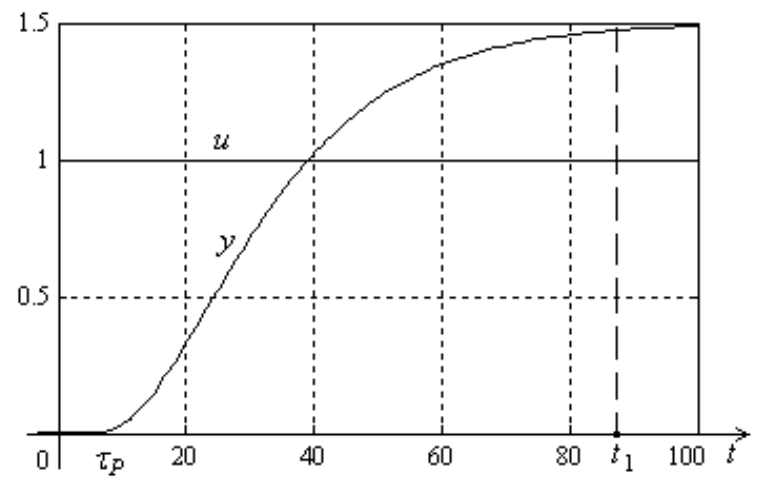

Figure 7. Process response to a unit step input 
The closed-loop system responses $y$ and $u$ to a unit step reference are illustrated in Figures 8 and 9 for $K=0.7 ; 1 ; 1.3 ; 2$. The control performance is suitable for $K=1.3$, too slow for $K=0.7$ and $K=1$, and too strong for $K=2$. The closed-loop system is stable for $K<5.35$.

Since the control response $u(t)$ for $K=1$ is close to a step shape (see Figure 9), it follows that the model parameters have been suitably chosen, and the model (10) of second-order with double lag time constant plus time delay describes the process sluggishness with sufficient accuracy.

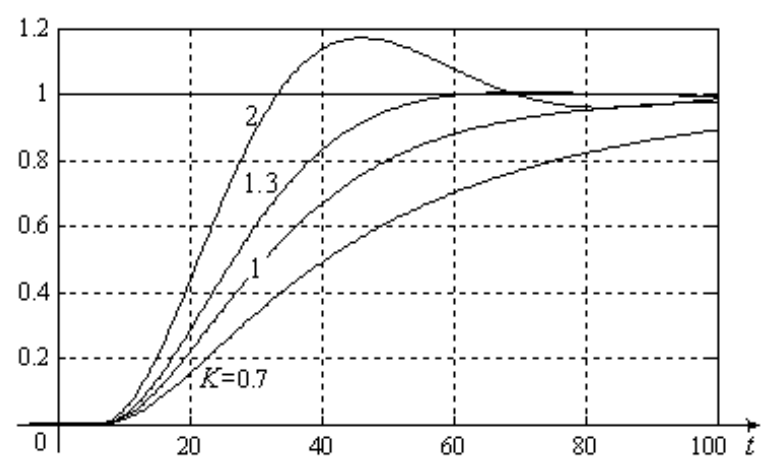

Figure 8. Responses $y(t)$ to a unit step reference for $K=0.7 ; 1 ; 1.3 ; 2$

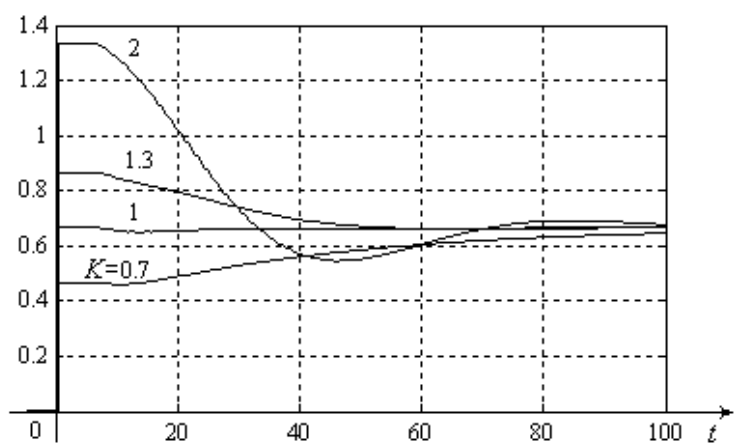

Figure 9. Responses $u(t)$ to a unit step reference for $K=0.7 ; 1 ; 1.3 ; 2$

Figures 10, 11 and 12 illustrate the closed-loop system responses $y(t)$ to a unit step reference for $K=1.3$ and different values of the model parameters. The responses show a high robustness of the control algorithm with respect to each of the parameters $K_{M}, \tau_{M}$ and $T_{t r M}$. The closed-loop system is respectively stable for $K_{M}>0.364$, for all $\tau_{M}>0$ and for $T_{t r M}>8.4$.

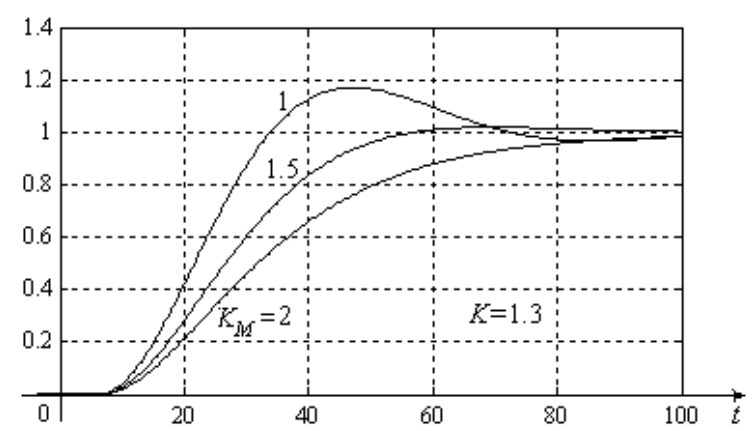

Figure 10. Responses $y(t)$ to a unit step reference for $\tau_{M}=9, T_{t r M}=80$ and $K_{M}=2 ; 1.5 ; 1$

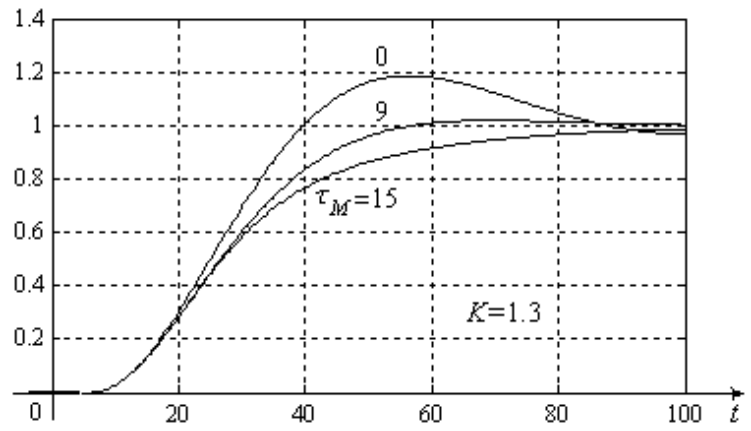

Figure 11. Responses $y(t)$ to a unit step reference for $K_{M}=1.5, T_{t r M}=80$ and $\tau_{M}=15 ; 9 ; 0$

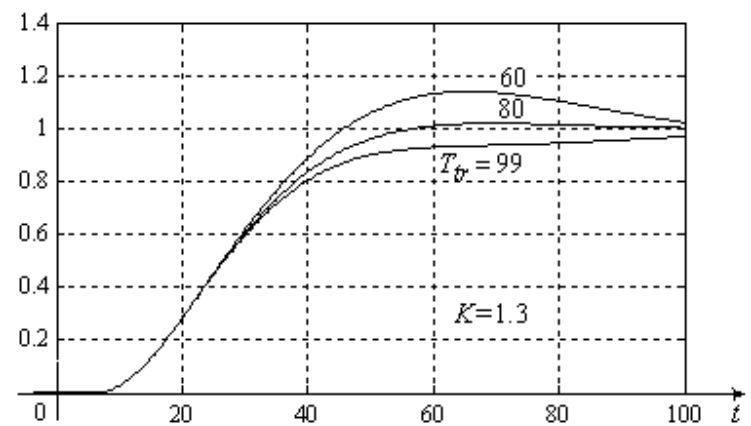

Figure 12. Responses $y(t)$ to a unit step reference for $K_{M}=1.5, \tau_{M}=9$ and $T_{t r M}=99 ; 80 ; 60$

Figures 13 and 14 illustrate the closed-loop system responses $y(t)$ for various values of the model parameters $\tau_{M}$ and $T_{t r M}$, and for the best values of the tuning parameter $K$. We see that the control performance remains high even for wrong settings of $\tau_{M}$ and $T_{t r M}$. For $K=1.8$ and the wrong $\tau_{M}=15$ (response A in Figure 13), the control performance is better than the one for $K=1$ and the wrong $\tau_{M}=5$ (response C), and even than the one for $K=1.3$ and the correct $\tau_{M}=9$ (response B). Also, for $K=1.67$ and the wrong $T_{t r M}=99$ (response A in Figure 14), as well as for 
$K=0.82$ and the wrong $T_{t r M}=65$ (response C), the control performance is comparable to the one for $K=1.3$ and the correct $T_{t r M}=80$ (response B). As a general recommandation, it is better to choose $\tau_{M}>\tau_{P}$ than $\tau_{M}<\tau_{P}$, and $T_{t r M}>T_{t r P}$ than $T_{t r M}<T_{t r P}$.

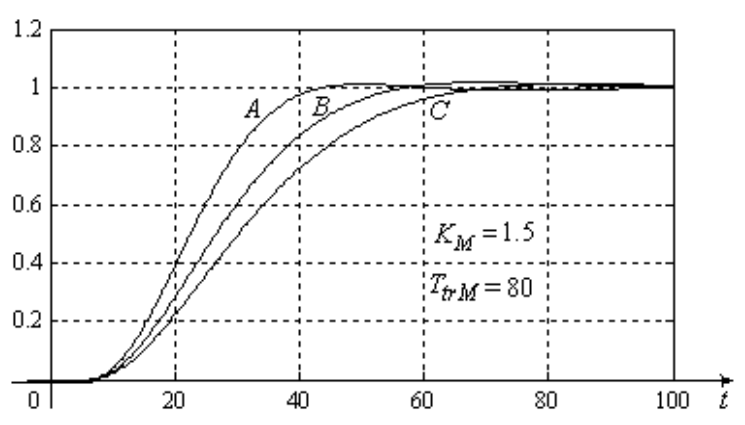

Figure 13. Responses $y(t)$ to a unit step reference for (A): $\tau_{M}=15, K=1.8$; (B): $\tau_{M}=9, K=1.3$; (C): $\tau_{M}=5, K=1$

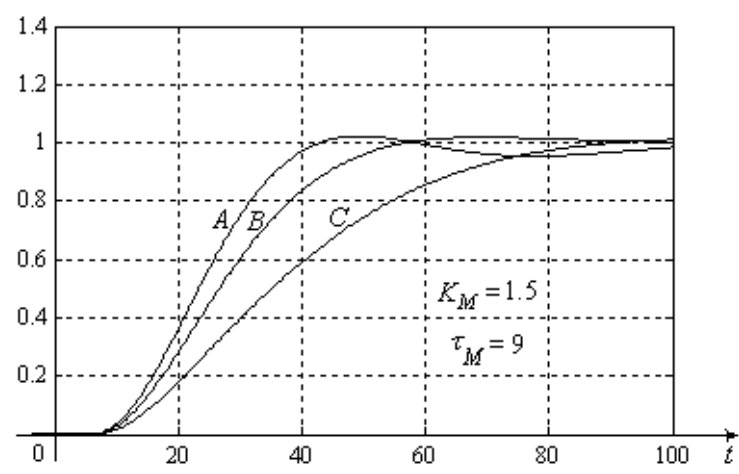

Figure 14. Responses $y(t)$ to a unit step reference for (A): $T_{t r M}=99, K=1.67$; (B): $T_{t r M}=80$, $K=1.3 ;(\mathrm{C}): T_{t r M}=65, K=0.82$

The control performance is better than the one achieved with a PI controller

$$
G_{P I}(s)=K_{R}\left(1+\frac{1}{T_{i} s}\right) .
$$

This follows from Figure 15, which shows the responses $y(t)$ to a unit step reference for $K_{R}=0.2,0.3,0.4$ and the best values of the integral time constant, namely $T_{i}=10.5,14,20$, respectively.

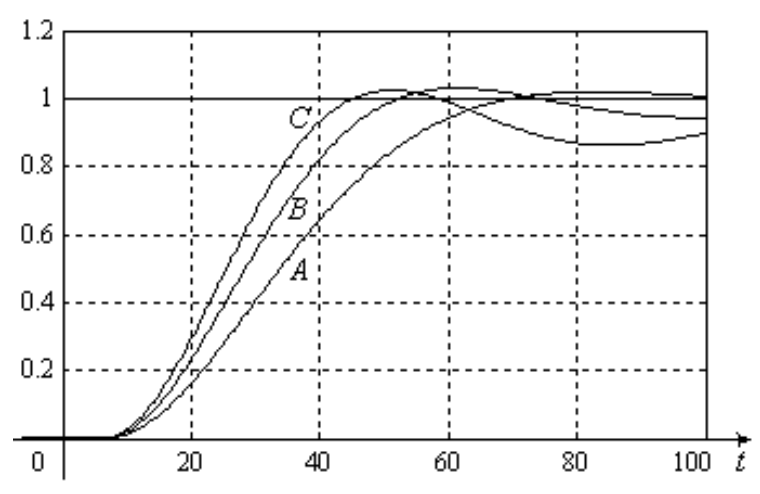

Figure 15. Responses $y(t)$ to a unit step reference for a PI controller with (A): $K_{R}=0.2, T_{i}=10.5$;

(B): $K_{R}=0.3, T_{i}=14$; (C): $K_{R}=0.4, T_{i}=20$

Application 2.2. Consider the proportional process of nonminimum phase

$$
G_{P}(s)=\frac{(-8 s+1) e^{-3 s}}{(5 s+1)(10 s+1)(15 s+1)} .
$$

From the process response to a unit step input in Figure 16, we get the following values of the model parameters:

$$
\begin{aligned}
& K_{M}=1, \quad \tau_{M}=18, \\
& T_{t r M}=t_{1}-\tau_{P} \approx 85-18=67
\end{aligned}
$$

The closed-loop system responses to a unit step reference are shown in Figures 17 and 18. A good control performance is achieved for $K=1.1$. The closed-loop system is stable for $K<3.19$. Since the control response $u(t)$ for $K=1$ is close to a step shape, the model (10) describes with sufficient accuracy the process sluggishness.

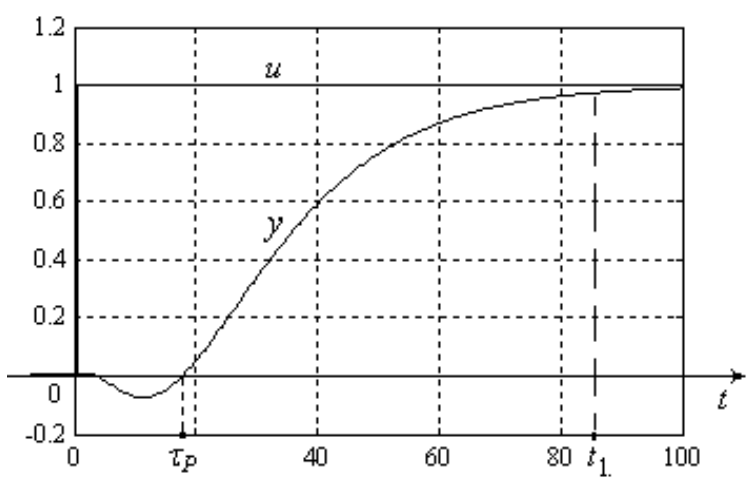

Figure 16. Process response to a unit step input 


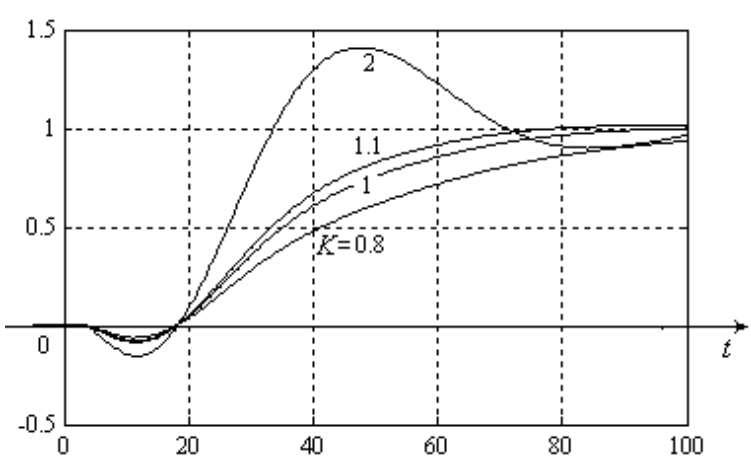

Figure 17. Responses $y(t)$ to a unit step reference for $K=0.8 ; 1 ; 1.1 ; 2$

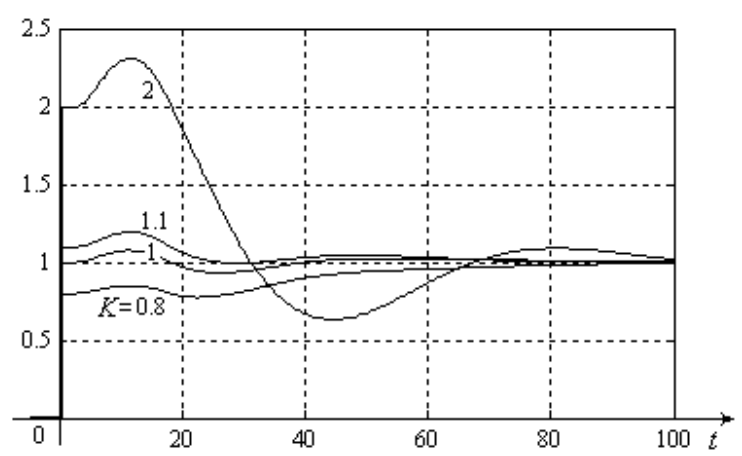

Figure 18. Responses $u(t)$ to a unit step reference for $K=0.8 ; 1 ; 1.1 ; 2$

Application 2.3. Consider the oscillatory process

$G_{P}(s)=\frac{1.5(2 s+1) e^{-6 s}}{(4 s+1)(5 s+1)\left(36 s^{2}+2.35 s+1\right)}$.

From the unit step process response in Figure 19, one gets

$K_{P}=1.5$,

$\tau_{P}=9$

$\sigma=33 \% \approx 1 / 3$,

$t_{0}=32$

therefore

$K_{M}=(1+\sigma) K_{P}=2$,

$\tau_{M}=9$

$T_{t r M}=t_{0}-\tau_{P}=32-9=23$

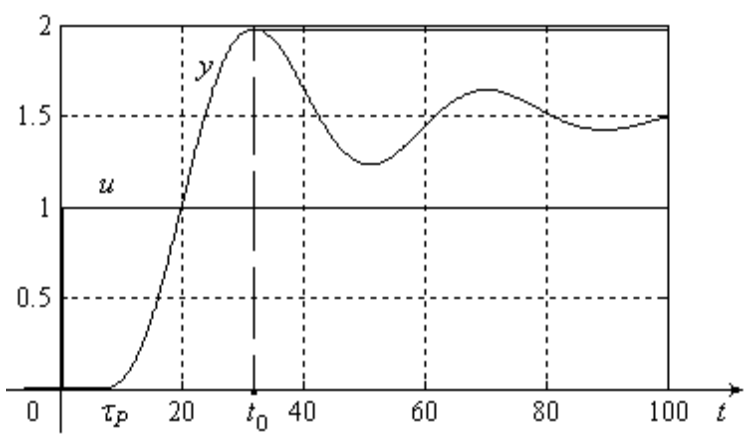

Figure 19. Process response to a unit step input

Figures 20 and 21 show the closed-loop system responses $y(t)$ and $u(t)$ to a unit step reference for $K=0.4, K=0.63$ and $K=1$. The transient response $y(t)$ for $K=0.63$ is sufficiently good for the given process. The closed-loop system is stable for $K<1.22$.

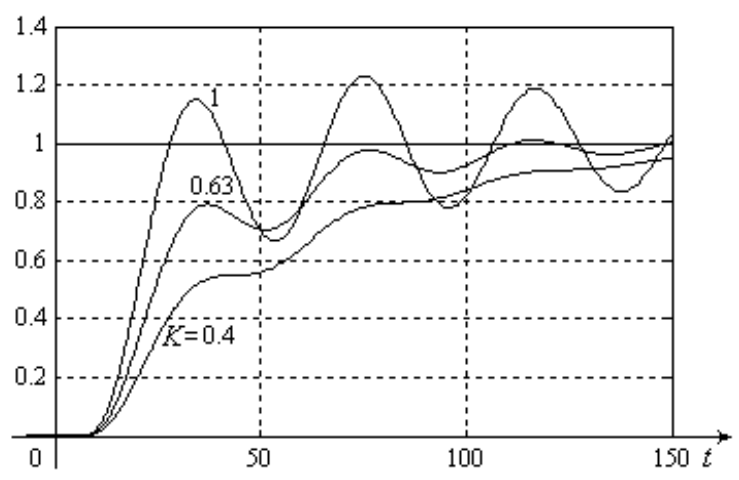

Figure 20. Responses $y(t)$ to a unit step reference for $K=0.4 ; 0.63 ; 1$

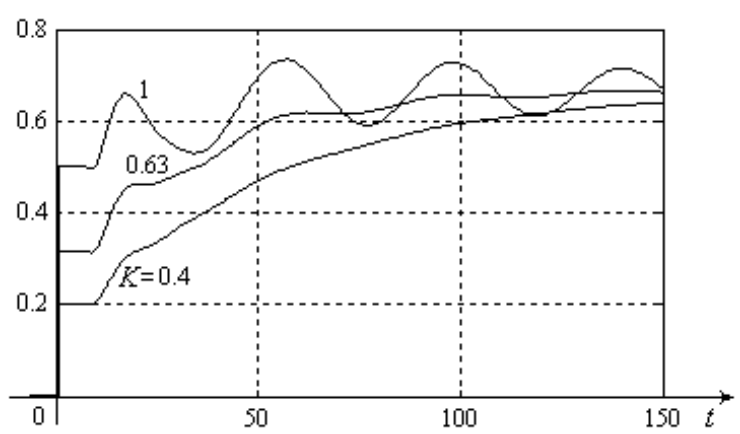

Figure 21. Closed-loop system responses $u(t)$ to a unit step reference for $K=0.4 ; 0.63 ; 1$

Application 2.4. For the non-oscillatory process with overshoot 


$$
G_{P}(s)=\frac{1.5(20 s+1) e^{-6 s}}{(4 s+1)(5 s+1)(6 s+1)},
$$

from the unit step response in Figure 22, we get

$K_{P} \simeq 1.5, \quad \tau_{P} \simeq 6$,

$$
\sigma \approx 49 \%=0.49, \quad t_{0}=18 \text {, }
$$

therefore

$$
\begin{aligned}
& K_{M}=(1+\sigma) K_{P} \approx 2.23, \quad \tau_{M}=6, \\
& T_{t r M}=t_{0}-\tau_{P}=18-6=12
\end{aligned}
$$

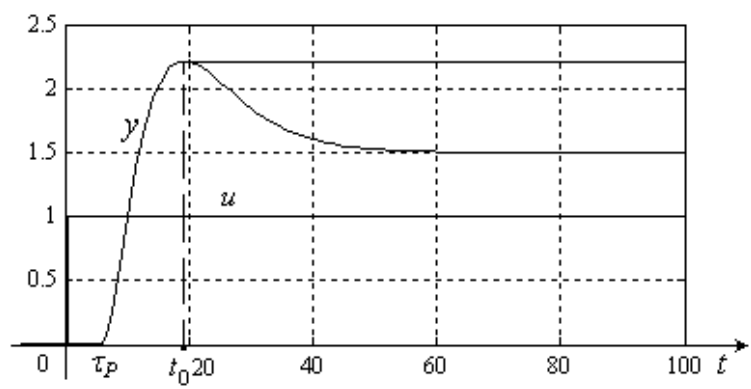

Figure 22. Process response to a unit step input

Figures 23 and 24 illustrate the closed-loop system responses $y$ and $u$ to a unit step reference for $K=1, K=0.88$ and $K=0.5$. The response $y(t)$ for $K=0.88$ is sufficiently fast for the given process. The closed-loop system is stable for $K<2.05$.

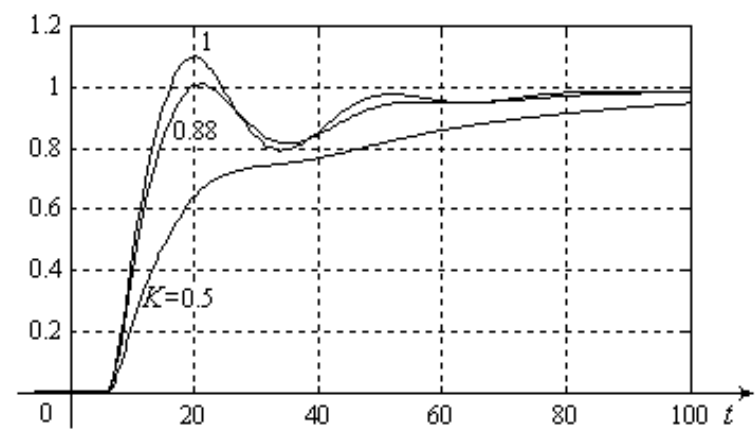

Figure 23. Responses $y(t)$ to a unit step reference for $K=0.5 ; 0.88 ; 1$

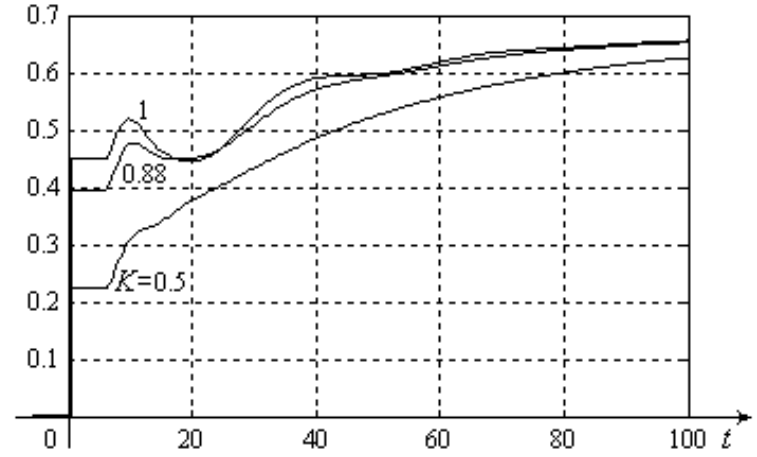

Figure 24. Closed-loop system responses $u(t)$ to a unit step reference for $K=0.5 ; 0.88 ; 1$

\section{Extended form of the control algorithm}

The proposed algorithm can be extended to control integral-type processes and some unstable processes.

\subsection{Theoretical basis}

The basic idea is to turn the original process into a stable proportional-type process (called compensated process), preferably without overshoot, by using a negative feedback path (see Figures 25 and 26).

As a rule, for an integral-type process, the step response of the compensated process is monotone and bounded for small values of the feedback gain $K_{f}$, becoming faster or even oscillatory by increasing the gain $K_{f}$. It is recommended to choose a large $K_{f}$, but not too large to cause a step response with overshoot of the compensated process. For a chosen $K_{f}$, the model parameters $K_{M}, \tau_{M}$ and $T_{t r M}$ will be determined from the compensated process response $y(t)$ to a step change of the input $c$. For $K=1$, if the the model parameters $K_{M}, \tau_{M}$ and $T_{t r M}$ have appropriate values, then the closed-loop system response $c(t)$ to a step reference is close to a step form. This feature offers the process operator the possibility to verify and correct online the model parameters of the compensated process.

The overall controller $\mathbf{C}$ in Figure 26 is a two degrees-of-freedom controller with three operating modes: AUTOMATIC, MANUAL and COMPENSATORY. In MANUAL and COMPENSATORY modes, the process operator 
can directly set any desired values for the input $u$ of the process and the input $c$ of the compensated process, respectively. If $K_{f}=0$, then the MANUAL and COMPENSA-TORY modes coincide. Before switching to COMPENSATORY mode, the variable $c$ needs to be automatically set to the value of $u$.

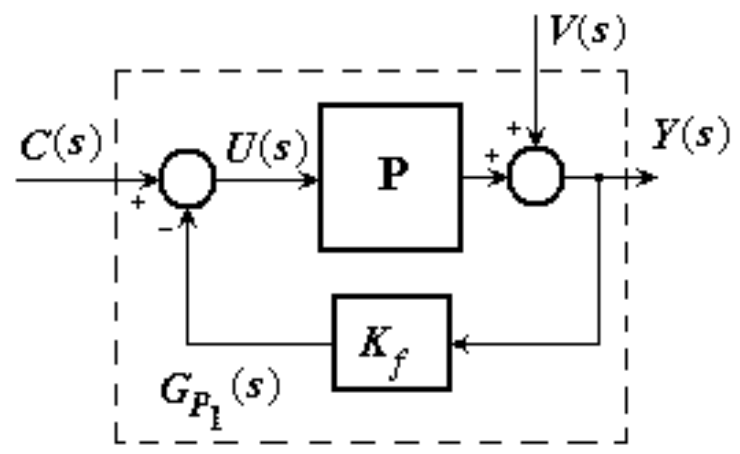

Figure 25. Block diagram of the compensated process

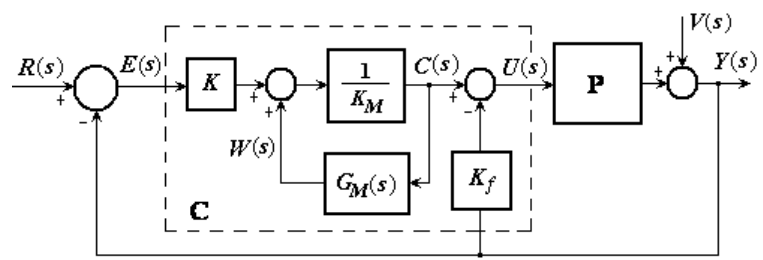

Figure 26. Closed-loop control system with compensated process

According to the controller structure in Figure 26 and the model transfer function (13), the discrete control algorithm has the following equations:

$\left\{\begin{array}{l}e_{k}=r_{k}-y_{k} \\ w_{k}=2 p w_{k-1}-p^{2} w_{k-2}+K_{M}(1-p)^{2} c_{k-l_{M}-1}, \\ c_{k}=\left(K e_{k}+w_{k}\right) / K_{M} \\ u_{k}=c_{k}-K_{f}\left(y_{k}-y_{0}\right)\end{array}\right.$

where $y_{0}$ is the value of $y$ before switching to AUTOMATIC mode, $l_{M}=\left[\tau_{M} / T\right]$ and

$p=e^{-a} \approx \frac{1}{1+a+a^{2} / 2+a^{3} / 6}, \quad a=5.83 T / T_{t r M}$.

For $K_{f}=0$, the extended control algorithm (20) reduces to the primary control algorithm. Let $u_{0}$ and $e_{0}$ be the values of $u$ and $e$ before switching to AUTOMATIC mode. To have a bumpless transfer only for error $e_{0}=0$, the following settings need to be made before switching to AUTOMATIC mode:

$c_{k-1}=c_{k-2}=\cdots=c_{k-l_{M}-1}=u_{0}$,
$w_{k-1}=w_{k-2}=K_{M} u_{0}$.

To have a bumpless transfer for any $e_{0} \neq 0$, it is needed to replace the third equation in (20) by

$c_{k}=\frac{K\left(e_{k}-e_{0}\right)+w_{k}}{K_{M}}$.

Remark 3.1. Consider a stable integral-type process with the transfer function

$G_{P}(s)=\frac{G_{1}(s)}{s}, \quad G_{1}(0)=K_{P 1} \neq 0$.

The compensated process in Figure 25 has the transfer functions

$G_{Y C}(s)=\frac{G_{P}(s)}{1+K_{f} G_{P}(s)}=\frac{G_{1}(s)}{s+K_{f} G_{1}(s)}$,

$G_{Y V}(s)=\frac{1}{1+K_{f} G_{P}(s)}=\frac{s}{s+K_{f} G_{1}(s)}$,

For the ramp disturbance $V(s)=1 / s^{2}$ and the input $C(s)=0$, the compensated process response is given by

$Y(s)=G_{Y V}(s) V(s)=\frac{1}{s\left[s+K_{f} G_{1}(s)\right]}$.

According to the final value theorem of the Laplace Transform, the response $y(t)$ has the final value

$y(\infty)=\lim _{s \rightarrow 0} s Y(s)=\frac{1}{K_{f} K_{P 1}}$.

Since the response $y(t)$ is finite, the steadystate error of the closed-loop system to a ramp disturbance (added to the process output) is zero.

\subsection{Simulation results}

In this subsection, we present two applications for two types of linear processes: a process of integral-type and an unstable process.

Application 3.1. Consider the integral-type process $G_{P}(s)=\frac{3(2 s+1) e^{-6 s}}{40 \mathrm{~s}(3 s+1)(4 s+1)}$.

Using a negative feedback path with $K_{f}<0.45$, the integral-type process turns into a compensated 
process whose response to a step input is monotone and bounded (see Figure 27). For $K_{f}=0.4$, the following model parameters can be deduced from the compensated process response: $K_{M}=2.5, \tau_{M}=7, T_{t r M}=t_{1}-\tau_{P}=84-7=77$

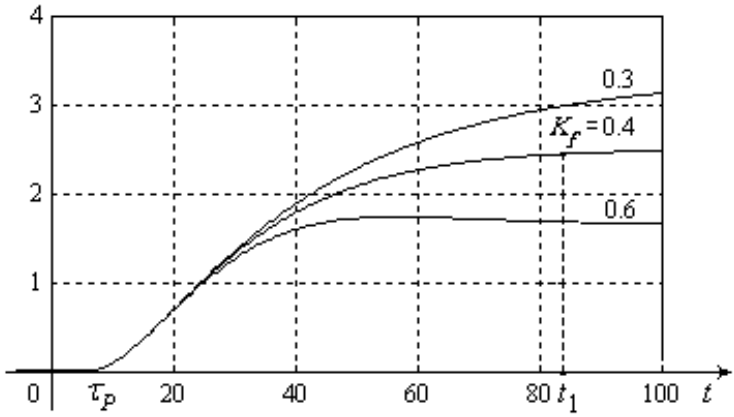

Figure 27. Responses $y(t)$ of the compensated process for $c=1(t)$ and $K_{f}=0.3 ; 0.4 ; 0.6$

The closed-loop system responses $y(t), c(t)$ and $u(t)$ to a unit step reference are shown in Figures 28,29 and 30 for $K=0.8 ; 1 ; 1.3 ; 2.2$. For $K=1.3$, the transient response $y(t)$ is very fast for the given integral process. The control system is stable for $K<6.35$.

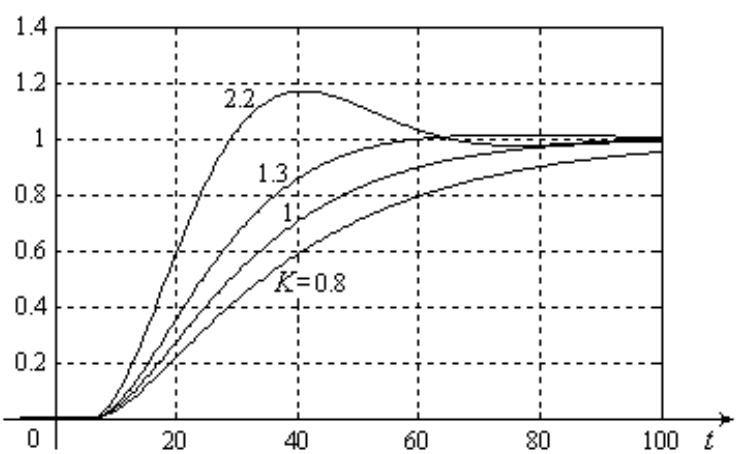

Figure 28. Responses $y(t)$ to a unit step reference for $K=0.8 ; 1 ; 1.3 ; 2.2$

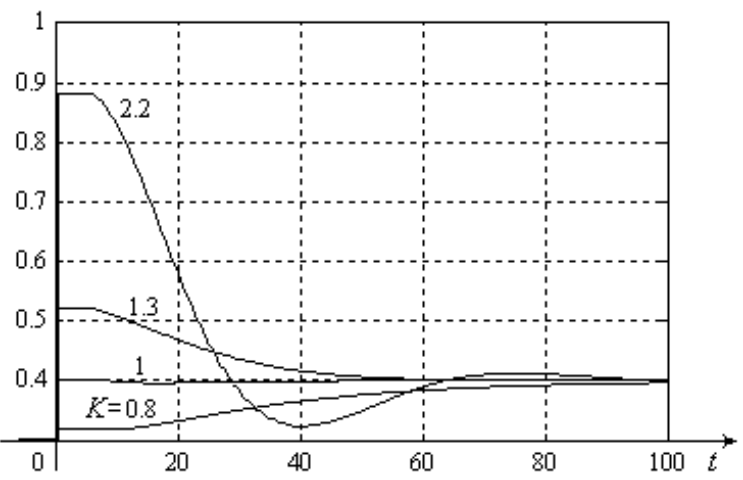

Figure 29. Closed-loop system responses $c(t)$ to a unit step reference for $K=0.8 ; 1 ; 1.3 ; 2.2$

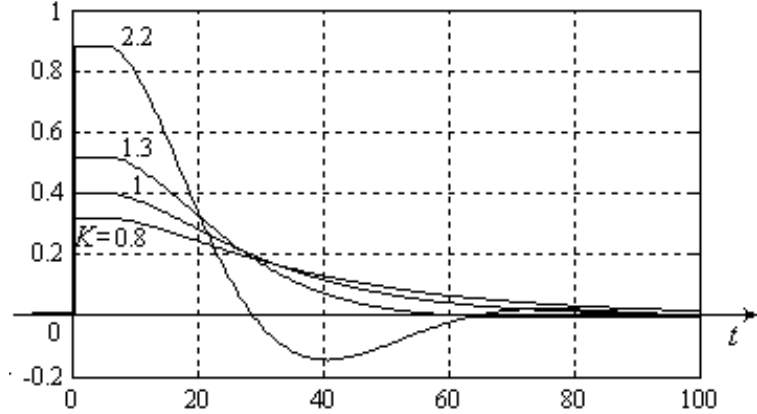

Figure 30. Closed-loop system responses $u(t)$ to a unit step reference for $K=0.8 ; 1 ; 1.3 ; 2.2$

For the suitable $K=1.3$, Figure 31 shows the closed-loop system responses $y(t)$ to a step and ramp disturbance. The steady-state error is zero for any step or ramp disturbance added to the process output.

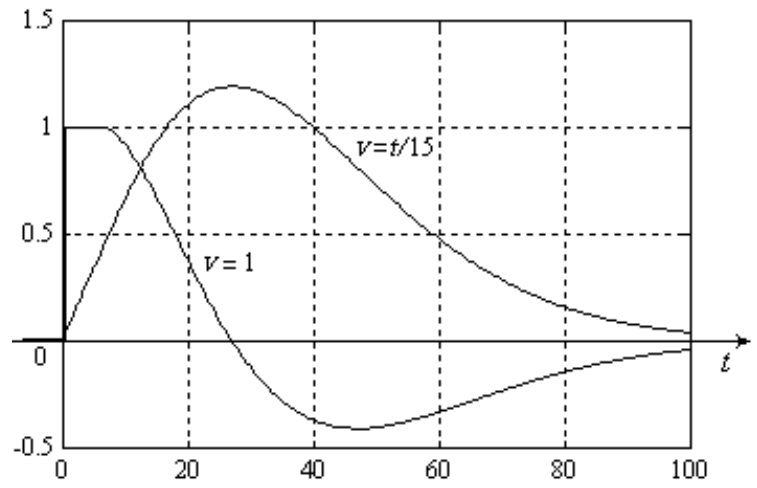

Figure 31. Closed-loop system responses $y(t)$ to a step and ramp disturbance

Application 3.2. Consider the unstable process

$G_{P}(s)=\frac{3 e^{-2 s}}{2(4 s+1)(10 s-1)}$.

Using a negative feedback path with $0.667<K_{f}<0.75$, the process turns into a stable compensated process whose response to a unit step input is monotone and bounded (see Figure 32). For $K_{f}=0.74$, the model parameters are:

$$
\begin{aligned}
& K_{M}=13.8, \\
& \tau_{M}=4, \\
& T_{t r M}=t_{1}-\tau_{P}=99-4=95
\end{aligned}
$$


The closed-loop system responses $y(t), c(t)$ and $u(t)$ to a unit step reference are shown in Figures 33,34 and 35 for $K=0.8 ; 1 ; 1.2$. The closedloop system is stable for $K<20.8$.

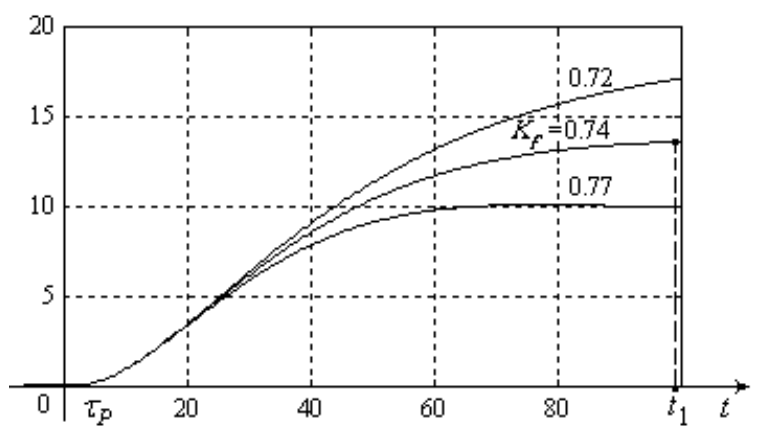

Figure 32. Compensated process responses $y(t)$ to the unit step input $c$

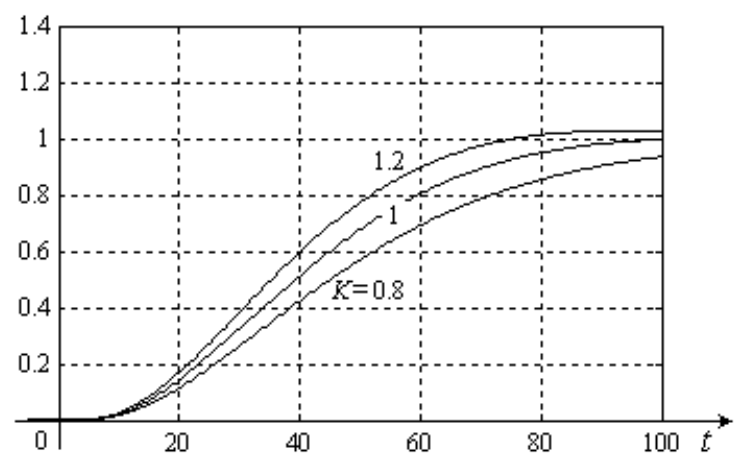

Figure 33. Responses $y(t)$ to a unit step reference for $K=0.8 ; 1 ; 1.2$

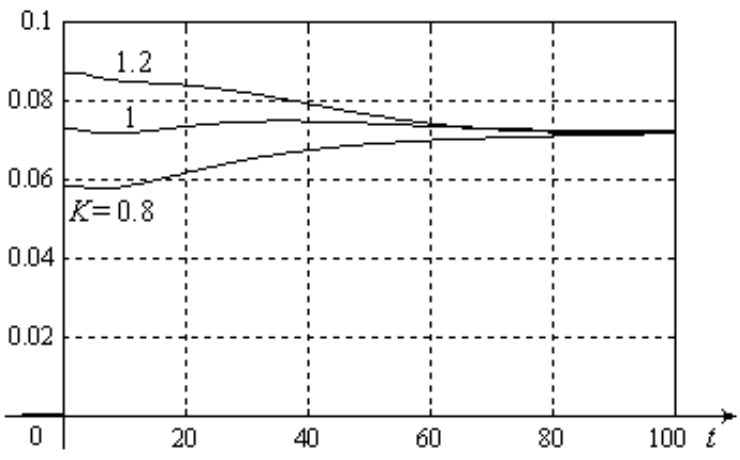

Figure 34. Closed-loop system responses $c(t)$ to a unit step reference for $K=0.8 ; 1 ; 1.2$

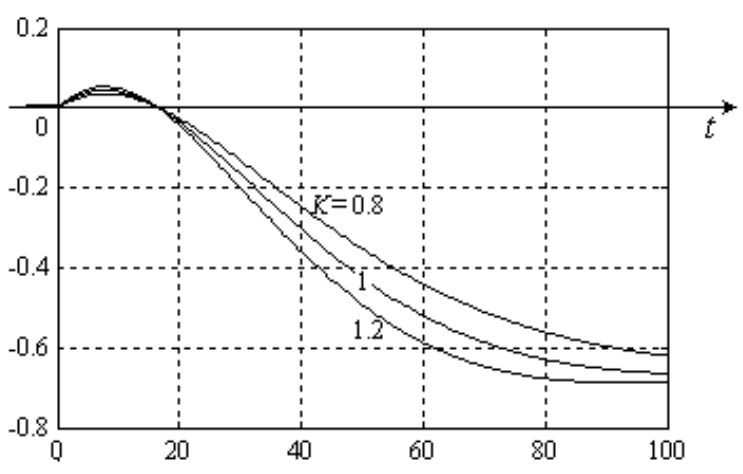

Figure 35. Closed-loop system responses $u(t)$ to a unit step reference for $K=0.8 ; 1 ; 1.2$

\section{Conclusions and future research}

Due to its capability to be used for almost all industrial processes, the proposed P-IMC control algorithm is an alternative to the PID and IMC algorithms. Actually, it is better than the PID algorithm with respect to both the control dynamic performance (especially for the processes with time delay) and the simplicity of the experimental tuning procedure. Also, it is more practical than IMC algorithms because it uses a single type of model for all categories of processes, and has a proportional gain $K$ as tuning parameter instead of a filter time constant.

In the primary (standard) variant, the algorithm can be used to control stable proportional processes (with or without time delay, overshoot and oscillations, of minimum or nonminimum phase). In this variant, the algorithm has four parameters: a tuning parameter $K$ that carries out the same function as the overall gain of the PID algorithm (used by the process human operator to increase or diminish the control action), and three model parameters that can be easily determined by experimental way - the model steady-state gain $K_{M}$, the model time delay $\tau_{M}$ and the model transient time $T_{t r M}$. In addition, there is a simple procedure to verify and correct online the model parameters, based on the fact that for an accurate model, the controller output to a step reference is close to a step form for $K=1$. This feature ensures and guaranties the stability of the closed-loop system on an upper bounded interval of $K$. Analyzing the deviation of the controller response to a step reference from the ideal step form, the process operator can adjust online the model parameters to improve the model accuracy. 
In its extended variant, the control algorithm has one more parameter, a process feedback gain $K_{f}$, which is used to control integraltype processes and some unstable processes, by turning them into stable proportional processes (compensated processes).

The proposed algorithm has been implemented in real time, in laboratory and some industrial applications, with excellent results.

In our opinion, this control algorithm can still be improved in order to reduce the weight of the

\section{REFERENCES}

1. Bengtsson, G. (1977). Output regulation and internal models - a frequency domain approach, Automatica, 13(4), 333-345.

2. Brosilow, C. \& Joseph, B. (2002). Techniques of Model-Based Control. Upper Saddle River (NJ), Prentice Hall.

3. Chia, T. L. \& Lefkowitz, I. (2010). Internal model-based control for integrating processes, ISA Transactions, 49(4), 519-527.

4. Cirtoaje, V. (2006). Process compensation based control, Buletinul Universitatii "Petrol-Gaze" din Ploiesti, Seria Tehnica, LVIII(1), 48-53.

5. Cirtoaje, V. \& Baiesu,A. (2016). Comparative study of three practical IMC algorithms with inner controller of first and second order, JEEE Control and Computer Science, 2(2), 21-28.

6. Cirtoaje, V. (2017). A quasi-universal practical IMC algorithm, Automatika, 58(2), $168-181$

7. Duma, R., Trusca, M. \& Dobra, P. (2011). Tuning and implementation of PID controllers using rapid control prototyping, J. of Control Engineering and Applied Informatics, 13(4), 64-73.

8. Francis, B. \& Wonham, W. (1976). The internal model principle of control theory, Automatica, 12(5), 457-465.

9. Garcia, C. \& Morari, M. (1982). Internal model control - A unifying review and some new results, Ind. Eng. Chem. Proc. Des. Dev., 21(2), 308-323. tuning gain $K$ on the control action, especially for the processes with large time delay, where the control response $c(t)$ to a unit step reference keeps its great initial value $K / K_{M}$ on the whole time interval $\left[0, \tau_{M}\right]$. This improvement can be achieved by adjusting the IMC component of the proposed P-IMC algorithm according to the procedure in [6], which allows to get a strictly monotone response $c(t)$ on $\left[0, \tau_{M}\right]$.

10. Vazquez-Guerra, R. J. et al. (2016). Dynamic delayed dontrollers for unstable recycling systems with time delays, Studies in Informatics and Control, 25(2), 195-206.

11. Ghousiya, B. K., Seshagiri, R. A. \& Radhakrishnan, T. K. (2017). Enhanced IMC based PID controller design for non-minimum phase integrating processes with time delay, ISA Transactions, 68, 223-234.

12. Horn, I., Arulandu, J., Gombas, C., VanAntwerp, J. \& Braatz, R. (1996). Improved filter design in internal model control, Ind. Eng. Chem. Res., 35, 3437-3441.

13. Jin, B. \& Liu, Q. (2014). Analytical IMC-PID design in terms of performance/robustness trade-off for integrating processes, $J$. Process Control, 24(12), 22-32.

14. Liu, T., Zhang, W. \& Gu, D. (2005). Analytical design of two-degree-of-freedom control scheme for open-loop unstable processes with time delay, J. Process Control, 15(5), 559-572.

15. Marlin, T. (1995). Process Control Designing processes and Control Systems for Dynamic Performance. New York, McGraw Hill, Inc.

16. Nageswara-Rao, C. V. \& Padma-Sree R. (2010). IMC based controllers design for integrating system with time delay, Indian Chem Eng, 52(3), 194-218.

17. Nguyen, T. H., Kaneko, O. \& Yamamoto, S. (2013). Fictitious reference iterative tuning to modified IMC for unstable plants, Journal of Control, Measurement, and System Integration, 6(5), 345-352. 
18. Nicolau, V. (2013). On PID controller design by combining pole placement technique with symmetrical optimum criterion, Mathematical Problems in Engineering, 1-8.

19. Normey-Rico, J. E. \& Camacho, E. F. (2007). Control of Dead-time Processes. SprigerVerlag London Ltd.

20. Rivera, D., Morari, M. \& Skogestad, S. (1986). Internal model control - PID controller design, Ind. Eng. Chem. Process Des. Dev., 25, 252-265.

21. Santosh-Kumar, D. B. \& Padma-Sree, R. (2016). Tuning OF IMC based PID controllers for integrating systems with time delay, ISA Transactions, 63, 242-255.

22. Saxena, S. \& Hote, Y. (2012). Advances in Internal Model Control Technique: A Review and Future Prospects, IETE Technical Review, 29(6), 461-472.

23. Singh, R., Bala, R. \& Bhatia, B. (2014). Internal Model Control and IMC Based
PID Controller, International Journal of Advanced Research in Computer Science and Software Engineering, 4(6), 915-922.

24. Vanavil, B., Anusha, A. V., Perumalsamy, M. \& Rao, A. S. (2014). Enhanced IMCPID controller design with lead-lag filter for unstable and integrating process with time delay, Chemical Engineering Communication, 201(11), 1468-1496.

25. Visioli, A. \& Zhong. Q. C. (2011). Control of Integral Processes with Dead Time. Springer Verlag London Ltd.

26. Yamada, K. \& Hagiwara, T. (2006). A design method of modified PID controller for unstable plant, Intelligent Engineering System Through Artificial Neural Networks, 16, 753-758.

27. Ziegler, J. G. \& Nichols, N. B. (1942). Optimum setting for automatic controllers, ASME Trans, 759-768. 